\title{
Electroproduction of Longitudinally Polarized Vector Mesons
}

\author{
Peter Kroll * \\ Universität Wuppertal, Fachbereich Physik \\ D-42097 Wuppertal, Germany
}

\begin{abstract}
It is reported on an analysis of electroproduction of light vector mesons at small Bjorken- $x\left(x_{\mathrm{Bj}}\right)$ within the handbag approach. The partonic subprocesses, meson electroproduction off quarks or gluons, are calculated within the modified perturbative approach (m.p.a.) in which quark transverse momenta are retained. The soft hadronic matrix elements, generalized parton distributions (GPDs), are constructed from the CTEQ6 parton distribution functions (PDFs) by means of double distributions. The cross sections for longitudinal polarized virtual photons evaluated from this approach, are in very good agreement with experiment in a wide range of kinematics.
\end{abstract}

It has been shown [1] that, at large photon virtuality $Q^{2}$, meson electroproduction factorizes in partonic subprocesses, electroproduction off gluons or quarks, $\gamma^{*} g(q) \rightarrow M g(q)$, and GPDs, representing soft proton matrix elements. It has also been shown that the dominant amplitude is that for transitions from longitudinally polarized virtual photons $\left(\gamma_{L}^{*}\right)$ to like-wise polarized vector mesons $\left(V_{L}\right)$. Other transitions are suppressed by inverse powers of $Q^{2}$. In the following it is reported on an analysis [2] of the process $\gamma_{L}^{*} p \rightarrow V_{L} p$, within this handbag factorization scheme carried through in the kinematical regime of low $x_{\mathrm{Bj}}(\lesssim 0.2)$.

The mentioned two partonic subprocesses lead to the following contributions to the amplitude $\mathcal{M}_{V}$ for the process $\gamma_{L}^{*} p \rightarrow V_{L} p:\left(x_{g}=0, x_{q}=-1\right)$

$$
\mathcal{M}_{V}^{g(q)}=e \sum_{a} e_{a} \mathcal{C}_{V}^{a} \int_{x_{g(q)}}^{1} d x \mathcal{H}_{V}^{g(q)}\left(x, \xi, Q^{2}, t=0\right) H^{g(a)}(x, \xi, t),
$$

which are to be summed coherently. The sum in (1) runs over all quark flavors while $e_{a}$ denotes the corresponding quark charges in units of the positron charge $e$. For $\rho$ and $\phi$ production the non-zero flavor weight factors $\mathcal{C}_{V}^{a}$ read $\mathcal{C}_{\rho}^{u}=-\mathcal{C}_{\rho}^{d}=1 / \sqrt{2}$ and $\mathcal{C}_{\phi}^{s}=1$, respectively. The amplitude (1) refers to proton helicity non-flip, the flip amplitude is neglected because our interest lies in small $-t$. The functions $H^{g(a)}$ represent GPDs for gluons and quarks. They are functions of three variables - $t$, a momentum fraction $x$ and skewness $\xi$. The latter one is kinematically fixed by $\xi \simeq x_{\mathrm{Bj}} /\left(2-x_{\mathrm{Bj}}\right)\left[1+m_{V}^{2} / Q^{2}\right]$ in a small $x_{\mathrm{Bj}}$ approximation $\left(m_{V}\right.$ being the mass of the vector meson). To vector meson electroproduction the GPD $\widetilde{H}$ and $\widetilde{E}$ do not contribute while $E$ can be ignored in the region of small $x_{\mathrm{Bj}}$ since it contributes $\propto \xi^{2}$ to the proton helicity non-flip amplitude. Thus, only the GPD $H$ is required. In (1) only its $t$-dependence is considered. That of the subprocess amplitude $\mathcal{H}_{V}$ provides power corrections of order $t / Q^{2}$ which are neglected. In the GPDs $t$ is scaled by a soft parameter, actually the slope of the diffraction peak.

The GPDs are constructed with the help of double distributions [3]. The chief advantage of this construction is the guaranteed polynomiality of the GPDs and the correct forward limit $\xi, t \rightarrow 0$. As is well-known at low $x$ the PDFs behave as powers $\delta_{i}$ of $x$. These powers are determined by the intercepts of appropriate Regge trajectories. The Regge behaviour

*The author thanks Markus Diehl for presenting this talk at the DIS07 conference. 
of the PDFs is transferred to the GPDs at $t=0$ by means of the double distribution construction. It seems plausible to generate the $t$-dependence of the GPDs by Regge ideas, too. Assuming linear Regge trajectories $\alpha_{i}(t)=\alpha_{i}(0)+\alpha_{i}^{\prime} t(i=\mathrm{g}$, sea, valence) and exponential $t$-dependencies of the Regge residues, one may employ the following ansatz for the double distribution $\left(n_{g}=n_{\text {sea }}=2, n_{\text {val }}=1\right)$

$$
f_{i}(\beta, \eta, t)=\mathrm{e}^{b_{i} t}|\beta|^{-\alpha_{i}^{\prime} t} h_{i}(\beta) \frac{\Gamma\left(2 n_{i}+2\right)}{2^{2 n_{i}+1} \Gamma^{2}\left(n_{i}+1\right)} \frac{\left[(1-|\beta|)^{2}-\eta^{2}\right]^{n_{i}}}{(1-|\beta|)^{2 n_{i}+1}},
$$

where the function $h_{i}$ represents a PDF suitably continued to negative values of $x$. The GPDs are then obtained by an integral over $f_{i}$

$$
H^{i}(x, \xi, t)=\int_{-1}^{1} d \beta \int_{-1+|\beta|}^{1-|\beta|} d \eta \delta(\beta+\xi \eta-x) f_{i}(\beta, \eta, t) .
$$

In Ref. [2] the Regge parameters are fixed in the following way: The integrated cross section $\sigma_{L} \sim \int d t\left|\mathcal{M}_{V}\right|^{2}$ behaves $\propto W^{\delta_{g}\left(Q^{2}\right)}$ at fixed $Q^{2}$ and small $x_{\mathrm{Bj}}$. Thus, $\delta_{g}$ can be fixed from the HERA data $[4,5,6]$. A fit provides $\delta_{g}=0.10+0.06 \ln \left(Q^{2} / 4 \mathrm{GeV}^{2}\right)$. For the slope of the gluon trajectory the value $\alpha_{g}^{\prime}=0.15 \mathrm{GeV}^{-2}$ is taken. Since the sea quarks mix with the gluons under evolution, $\alpha_{\text {sea }}(t)=\alpha_{g}(t)$ is assumed. For the valence quarks, on the other hand, a standard Regge trajectory is taken $-\alpha_{\text {val }}=0.48+0.90 \mathrm{GeV}^{-2} t$. The slope parameter of the gluon and sea quark Regge residue is obtained from a fit to the HERA data on the differential cross section $[4,6]: b_{g}=b_{\text {sea }}=2.58 \mathrm{GeV}^{-2}+0.25 \mathrm{GeV}^{-2} \ln \left[m^{2} /\left(Q^{2}+m^{2}\right)\right]$ ( $m$ being the proton's mass). In the zero skewness limit the valence quark GPDs read

$$
H_{\text {val }}^{q}(x, \xi=0, t=0)=\mathrm{e}^{b_{\text {val }} t} q_{\text {val }}(x) .
$$

This is very close to the ansatz advocated for in Ref. [7] in order to extract the zero-skewness GPDs from the nucleon form factor data. The comparison with that analysis reveals that one may choose $b_{\text {val }}=0$.

Working out the subprocess amplitudes from the relevant Feynman graphs in collinear approximation and to LO, one arrives at the following amplitude for $\rho$ production

$$
\mathcal{M}_{\rho}=e \frac{8 \pi \alpha_{s}}{N_{c} Q} f_{\rho}\langle 1 / \tau\rangle_{\rho} \frac{1}{\sqrt{2}}\left\{\frac{1}{2 \xi} I_{g}+\kappa_{s} C_{F} I_{\text {sea }}+\frac{1}{3} C_{F} I_{\text {val }}^{u}+\frac{1}{6} C_{F} I_{\text {val }}^{d}\right\} .
$$

The integral $I_{g}$ reads

$$
I_{g}=2 \int_{0}^{1} d x \frac{\xi H^{g}(x, \xi, t)}{(x+\xi)(x-\xi+i \epsilon)} .
$$

Analogous expressions hold for the other two integrals. For $\phi$ production the decay constant $f_{\rho}$ and the $1 / \tau$ moment of the $\rho$ distribution amplitude are to be replaced by the corresponding quantities for the $\phi$ meson. The charge factor $1 / \sqrt{2}$ is to be replaced by $-1 / 3$ and there is no valence quark contribution. For simplicity it is assumed that the $u$ and $d$ sea quark GPDs are proportional to that of the strange quark. The factor of proportionality, $\kappa_{s}$, is obtained from the CTEQ6 PDFs. It is about 2 at $Q^{2}=4 \mathrm{GeV}^{2}$ and tends towards 1 for increasing $Q^{2}$. Evaluating the GPDs from the CTEQ6M PDFs [8] and adopting the asymptotic $\rho$ meson distribution amplitude, leading to $\langle 1 / \tau\rangle=3$, one can work out the cross section $\sigma_{L}$ for $\rho$ production. The result, shown in Fig. 1, is evidently too large by order of magnitude at low $Q^{2}$. The deviations diminish with increasing $Q^{2}$. Note that there are large NLO corrections [9] which cancel the LO term to a large extent. Wether the inclusion of higher orders lead to agreement with experiment is unknown as yet. 
As is well-known from studies of the large momentum transfer behaviour of electromagnetic form factors, the collinear approximation becomes inconsistent in the end-point regions, since the contributions from large transverse separations, $\mathbf{b}$, of the quark and antiquark forming the meson are not sufficiently suppressed. In order to eliminate that defect the so-called m.p.a. has been invented [10] in which quark transverse degrees of freedom are retained and the accompanying gluon radiation ( a Sudakov factor) is taken into account. This m.p.a. is employed in the calculation of the partonic subprocesses. Instead of distribution amplitudes rather meson wave functions have to be used in this approach. Actually, a Gaussian one $\sim \exp \left[-a_{V}^{2} k_{\perp}^{2} /(\tau(1-\tau))\right]$ is utilized in [2]. The transverse size parameters are considered as free parameters to be adjusted to the experiment $\left(a_{\rho}=0.75 \mathrm{GeV}^{-1}\right.$, $\left.a_{\phi}=0.70 \mathrm{GeV}^{-1}\right)$. The structure of the amplitude as given in Eq. (2) still holds if the m.p.a. is used, even the relative strength of the various contributions remain to be the same.

Detailed comparison with experiment is made in Ref. [2]. Here, only a few results are presented. As one can see from Fig. 1 if the m.p.a. is used, the handbag result for $\sigma_{L}(\rho)$ is in fair agreement with the HERA data $[4,5]$ in particular if one considers the uncertainties in the theoretical results due to the errors of the PDFs. Results for $\sigma_{L}$ at lower values of $W$ are shown in Fig. 2 . Next, in Fig. 3 the energy dependence of $\sigma_{L}(\rho)$ is displayed. The figure also reveals the prominent role of the gluonic contribution. The valence quark contribution is only significant below $10 \mathrm{GeV}$. Results of similar quality are obtained for $\phi$ production. Here, only the ratio of the cross section for $\phi$ and $\rho$ production is shown in Fig. 4 . For $Q^{2} \rightarrow \infty$ the handbag approach predicts $\sigma_{L}(\phi) / \sigma_{L}(\rho) \longrightarrow 2 / 9\left(f_{\phi} / f_{\rho}\right)^{2}=0.248$. The deviations from this limit seen in Fig. 4 at finite $Q^{2}$, are generated by the breaking of flavor symmetry in the sea and, although to a lesser extent, by the meson wave function. The low value of the ratio at $W=5 \mathrm{GeV}$ is due to the additional valence quark contribution to the $\rho$ cross section.

In summary - the handbag factorization scheme with the partonic subprocesses calculated within the m.p.a. and GPDs constructed from the double distributions provides reasonable results for the longitudinal cross section of $\rho$ and $\phi$ electroproduction in a large range of $Q^{2}$ 
and $W$. As shown in Ref. [13] this approach can also be applied to the amplitude for transversally polarized photons. The infrared singularities occuring for that amplitude in collinear approximations are regularized in the m.p.a. by the quark transverse momenta. In Ref. [13] this amplitude has been calculated for HERA kinematics assuming that only the gluonic subprocess contributes. The inclusion of quarks for this amplitude is in progress. This analysis will provide results on $\sigma_{T}$ and on various spin density matrix elements for the same range of $Q^{2}$ and $W$ as for $\sigma_{L}$.

\section{References}

[1] A.V. Radyushkin, Phys. Lett. B385, 333 (1996); J.C. Collins et al., Phys. Rev. D56, 2982 (1997).

[2] S. V. Goloskokov and P. Kroll, Eur. Phys. J. C 50, 829 (2007).

[3] D. Müller et al, Fortschr. Phys. 42, 101 (1994); A.V. Radyushkin, Phys. Lett. B449, 81 (1999).

[4] C. Adloff et al., [H1 collaboration], Eur. Phys. J. C13, 371 (2000).

[5] J. Breitweg et al., [ZEUS collaboration], Eur. Phys. J. C6, 603 (1999).

[6] S. Chekanov et al., [ZEUS collaboration], Nucl. Phys. 718, 3 (2005).

[7] M. Diehl, T. Feldmann, R. Jakob and P. Kroll, Eur. Phys. J. C39, 1 (2005).

[8] J. Pumplin et al, JHEP 0207, 012 (2002).

[9] D. Y. Ivanov et al. JETP Lett. 80, 226 (2004) [Pisma Zh. Eksp. Teor. Fiz. 80, 255 (2004)], M. Diehl und W. Kugler, these proceedings.

[10] J. Botts and G. Sterman, Nucl. Phys. B325, 62 (1989).

[11] A. Airapetain et al., [HERMES collaboration], Eur. Phys. J. C17, 389 (2000).

[12] M.R. Adams et al., [E665 collaboration], Z. Phys. C74, 237 (1997).

[13] S.V. Goloskokov and P. Kroll, Eur. Phys. J. C42 281 (2005).

[14] Slides:

http://indico. cern. ch/contributionDisplay $\cdot$ py? contribId=77\&sessionId=7\&conf $I d=9499$

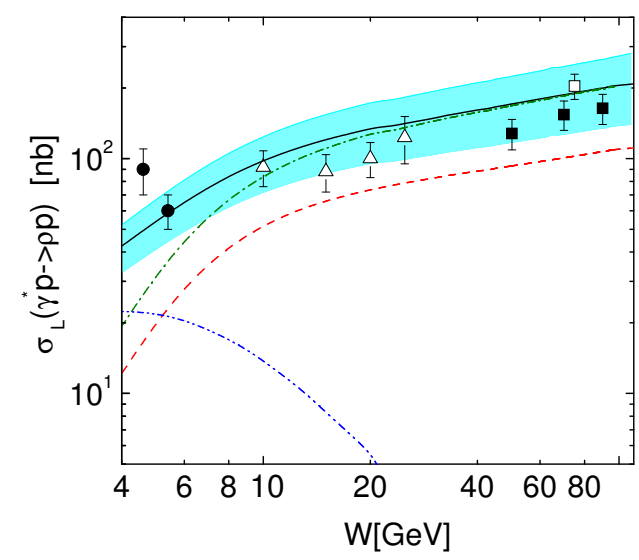

Figure 3: $\sigma_{L}(\rho)$ at $Q^{2}=4 \mathrm{GeV}^{2}$. Data taken from H1 [4] (solid square), ZEUS [5] (open square), E665 [12] (open triangle) and HERMES [11] (solid circle). The dashed (dashdotted, dash-dot-dotted) line represents the gluon (gluon+sea, (gluon+sea)-valence interference plus valence quark) contribution. For further notation, cf. Fig. 1.

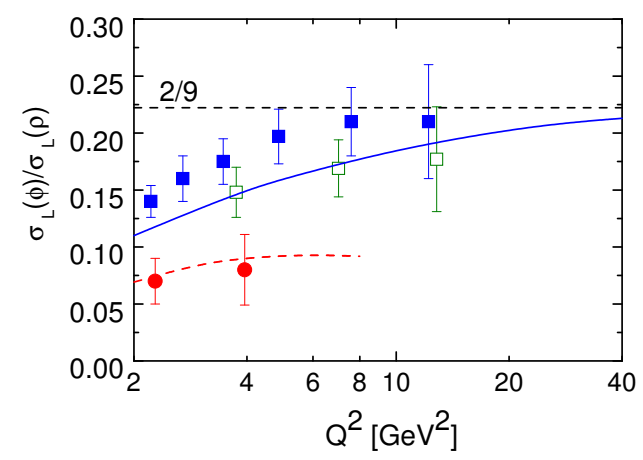

Figure 4: The ratio $\sigma_{L}(\phi) / \sigma_{L}(\rho)$. The solid (dashed) line represents the handbag result at $W=75(5) \mathrm{GeV}$. Data are taken from $\mathrm{H} 1$, ZEUS and HERMES. 